\title{
A near-infrared fluorophore for live-cell super- resolution microscopy of cellular proteins
}

\author{
Gražvydas Lukinavičius ${ }^{1 \dagger}$, Keitaro Umezawa ${ }^{1 \dagger}$, Nicolas Olivier ${ }^{2}$, Alf Honigmann ${ }^{3}$, Guoying Yang ${ }^{4}$, \\ Tilman Plass ${ }^{5}$, Veronika Mueller ${ }^{3}$, Luc Reymond', Ivan R. Corrêa Jr6, Zhen-Ge Luo7, Carsten Schultz ${ }^{5}$, \\ Edward A. Lemke ${ }^{5}$, Paul Heppenstall ${ }^{4}$, Christian Eggeling ${ }^{3,8}$, Suliana Manley ${ }^{2}$ and Kai Johnsson ${ }^{1 \star}$
}

\begin{abstract}
The ideal fluorescent probe for bioimaging is bright, absorbs at long wavelengths and can be implemented flexibly in living cells and in vivo. However, the design of synthetic fluorophores that combine all of these properties has proved to be extremely difficult. Here, we introduce a biocompatible near-infrared silicon-rhodamine probe that can be coupled specifically to proteins using different labelling techniques. Importantly, its high permeability and fluorogenic character permit the imaging of proteins in living cells and tissues, and its brightness and photostability make it ideally suited for live-cell super-resolution microscopy. The excellent spectroscopic properties of the probe combined with its ease of use in live-cell applications make it a powerful new tool for bioimaging.
\end{abstract}

\lceil lexible and specific methods to couple synthetic fluorescent probes to proteins in living cells are established, yet these methods are not generally compatible with the fluorophores best suited for live-cell imaging ${ }^{1}$. Fluorescent labels that are excited and emit in the near-infrared are especially biocompatible, because they avoid the use of light, which may cause phototoxicity or unwanted autofluorescent background ${ }^{2}$. Although numerous synthetic near-infrared fluorophores with exceptional photostability and brightness exist, they tend to be membrane impermeable and show unspecific binding to cellular components (Supplementary Table S1 and Fig. S1) $)^{3}$. Thus, invasive approaches, such as electroporation or glass-bead loading, are required to introduce these probes into cells and/or cumbersome additional manipulations are needed to reduce the background signal from unspecific binding ${ }^{4-6}$. Here, we introduce a highly permeable and biocompatible near-infrared silicon-rhodamine ( $\mathrm{SiR}$ ) fluorophore that can be coupled specifically to intracellular proteins in live cells and tissues using different labelling techniques. The fluorogenic character of the probe and its high brightness permit live-cell imaging experiments without washing steps. In addition, the fluorophore proved to be ideally suited for live-cell super-resolution microscopy.

\section{Results and discussion}

To develop near-infrared fluorophores suitable for the specific labelling of proteins inside living cells we turned our attention to a recently introduced class of fluorophores based on silicon-containing rhodamine derivatives such as SiR-methyl (Fig. 1a) ${ }^{7-9}$. SiR derivatives were shown to possess excellent spectroscopic properties, and in certain cases to be membrane permeable ${ }^{8}$. To achieve selective coupling of $\mathrm{SiR}$ derivatives to the proteins of interest, we envisioned exploiting self-labelling protein tags such as SNAP-tag ${ }^{1}$. SNAP-tag fusion proteins can be labelled specifically with molecular probes using benzylguanine (BG) derivatives ${ }^{10,11}$. However, imaging experiments with a BG derivative of SiR-methyl revealed an unacceptably high background signal from unspecific binding of the probe to different cellular structures (Supplementary Fig. S2). We speculated that exchange of the methyl group at the 2-position of the phenyl ring by a carboxyl group would reduce the hydrophobicity and unspecific binding of the fluorophore. At the same time the carboxyl group would permit the molecule to be in equilibrium with the uncharged spirolactone and could increase membrane permeability (Fig. 1a). Recently, spirolactames and spirolactones of $\mathrm{SiR}$ derivatives were described for the development of an irreversible turn-on sensor for $\mathrm{Hg}^{+}$(ref. 12).

We therefore synthesized SiR-carboxyl in five steps from commercially available material in an overall yield of $22 \%$ (for details see the Supplementary Information). SiR-carboxyl is excitable at around 640-650 nm and emits at around 660-670 nm, and the excitation and emission maxima were found to be $645 \mathrm{~nm}$ and $661 \mathrm{~nm}$, respectively (Supplementary Fig. S3a). It possesses an extinction coefficient of $100,000 \mathrm{M}^{-1} \mathrm{~cm}^{-1}$ and a fluorescence quantum yield of 0.39 in aqueous solution (Supplementary Table S2). An important difference between SiR-carboxyl and tetramethylrhodamine (TMR, Fig. 1a) is the higher propensity of SiR-carboxyl to form the non-fluorescent spirolactone in solvents of low dielectric constant $\varepsilon$, which we investigated by using dioxane-water mixtures $^{13}$. SiR-carboxyl exists predominantly in its spirolactone form in dioxane-water mixtures of dielectric constant less than 30, conditions under which TMR remains predominantly in its open form (Fig. 1b and Supplementary Fig. S3b). As expected, SiR-methyl is not sensitive to changes in solvent polarity as it cannot form a spirolactone (Fig. 1b). Next, we synthesized derivatives of SiR-carboxyl for the covalent labelling of the SNAP-tag, as well as for CLIP-tag and Halo-tag fusion proteins (dubbed

'Ecole Polytechnique Fédérale de Lausanne, Institute of Chemical Sciences and Engineering (ISIC), Institute of Bioengineering, National Centre of Competence in Research (NCCR) in Chemical Biology, 1015 Lausanne, Switzerland, ${ }^{2}$ Ecole Polytechnique Fédérale de Lausanne, Laboratory of Experimental Biophysics, NCCR in Chemical Biology, 1015 Lausanne, Switzerland, ${ }^{3}$ Max-Planck-Institute for Biophysical Chemistry, Department NanoBiophotonics, Am Fassberg 11, 37077 Göttingen, Germany, ${ }^{4}$ European Molecular Biology Laboratory, Mouse Biology Unit, via Ramarini 32, 00015 Monterotondo (RM), Italy, ${ }^{5}$ European Molecular Biology Laboratory, Meyerhofstrasse 1, 69117 Heidelberg, Germany, ${ }^{6}$ New England Biolabs Inc., 240 County Road, Ipswich, Massachusetts 01938, USA, Institute of Neuroscience and State Key Laboratory of Neuroscience, Shanghai Institutes for Biological Sciences, Chinese Academy of Sciences, Shanghai 200031, China, ${ }^{8}$ Medical Research Council Human Immunology Unit, Weatherall Institute of Molecular Medicine, University of Oxford, UK; ${ }^{\top}$ These authors contributed equally to this work. ${ }^{\star}$ e-mail: kai.johnsson@epfl.ch 


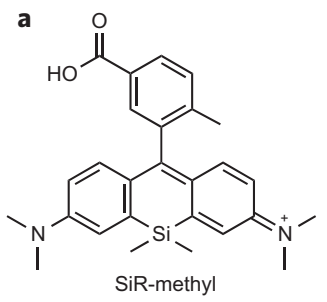<smiles></smiles><smiles></smiles><smiles>[R]C(=O)c1ccc2c(c1)C1(OC2=O)c2ccc(N(C)C)cc2[Si](C)(C)c2cc(N(C)C)ccc21</smiles>

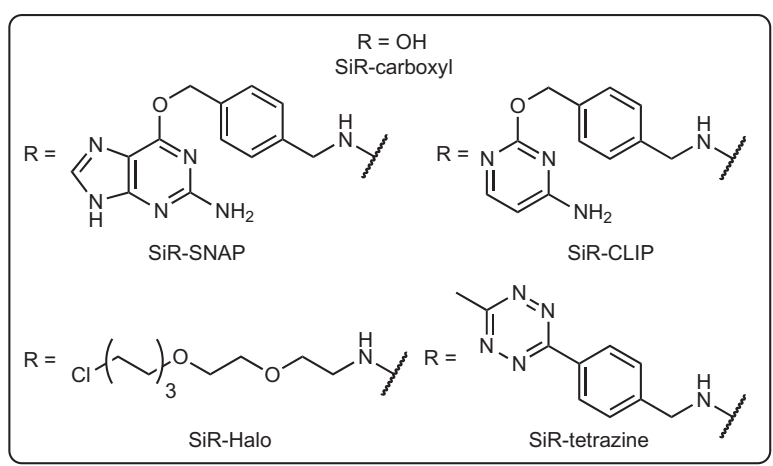

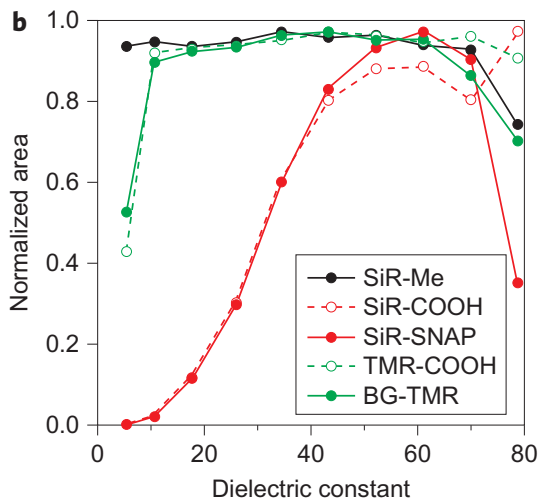

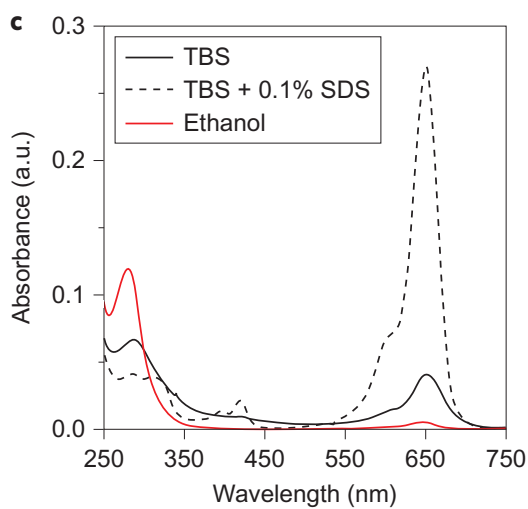

Figure 1 | SiR dyes used for SNAP-, CLIP-, Halo-tag and tetrazine labelling. a, Structures of SiR dyes, TMR and the formation of spirolactone of SiR-carboxyl. b. Normalized integral of absorption spectra of the zwitterion region of SiR and TMR derivatives in water-dioxane mixtures as a function of dielectric constant. Note that absorbance at $\varepsilon=80$ ( $0 \%$ of dioxane) is affected by the aggregation of fluorophores. BG-TMR represents TMR coupled to BG (Supplementary Fig. S2) $)^{11}$. c, Absorption spectra of $2.5 \mu \mathrm{M}$ SiR-SNAP measured in ethanol (red), Tris-buffered saline (TBS) buffer with (dashed black) and without (solid black) $0.1 \%$ sodium dodecyl sulfate (SDS).

SiR-SNAP, SiR-CLIP and SiR-Halo; Fig. 1a). CLIP-tag and Halotag are two other popular protein tags utilized for the covalent labelling of proteins in living cells ${ }^{14,15}$. All three tags can be labelled readily with their corresponding substrates, with rate constants comparable to those reported for other substrates (Supplementary Table S2). Furthermore, the relative fluorescence quantum yield of the protein-conjugated dye is comparable to that of SiR-carboxyl (Supplementary Table S2). SiR-labelled proteins also possess excellent photostability: the bleaching rate of the SiR-SNAP-labelled SNAP-tag is identical to those of Atto647N-labelled proteins (Atto647N is a dye frequently used in single-molecule applications) and much lower than the bleaching rate of Alexa647-labelled proteins (Supplementary Fig. S3c).

Live-cell imaging with SiR-carboxyl derivatives. We applied SiR-SNAP, SiR-CLIP and SiR-Halo for live-cell imaging. To demonstrate the potential of SiR-labelled proteins for multicolour imaging, these experiments were performed in a HeLa cell line that stably expressed a green fluorescence protein (GFP) fusion with $\alpha$-tubulin (GFP- $\alpha$-tubulin) and a mCherry fusion with histone $\mathrm{H} 2 \mathrm{~B}$ (H2B-mCherry) as markers ${ }^{16}$. We observed a specific fluorescence labelling of fusion proteins of SNAP-, CLIP- and Halo-tags in different organelles of living cells within 30-60 minutes, with no significant background signal (Fig. 2), which demonstrates that SiR-SNAP, SiR-CLIP and SiR-Halo readily pass through the plasma membrane as well as the internal membranes. An important feature of the dye for cellular applications is that the reactions of SiR-SNAP, SiR-CLIP and SiR-Halo in a cell culture medium (DMEM) containing 10\% fetal bovine serum (FBS) with their cognate protein-tag results in a more than fivefold increase in the fluorescence signal (Supplementary Fig. S5a). Consequently, fluorescence imaging of SiR-labelled proteins can be performed, albeit with some background signal, even without removing excess substrate through a washing step (Supplementary Fig. S4). The highly efficient and selective fluorescence labelling of intracellular proteins with SiR-SNAP, SiR-CLIP and SiR-Halo sets the dye apart from all the other near-infrared fluorophores that we tested (Supplementary Table S1).

Why are SiR-carboxyl derivatives so exceptionally well suited for live-cell labelling? A possible explanation is that the reactions of SiR-SNAP, SiR-CLIP and SiR-Halo with their respective protein tags keeps the fluorophores in their fluorescent zwitterionic form, and the aggregation of unreacted dye and unspecific binding to hydrophobic structures, two sources of background signal when using synthetic fluorophores in live cells, results in the formation of non-fluorescent spirolactone. This hypothesis is based on the following observations: addition of the surfactant SDS to aqueous solutions of SiR-SNAP, SiR-CLIP and SiR-Halo increased their fluorescence intensity more than threefold, which indicates their reversible aggregation and the concomitant formation of the nonfluorescent spirolactone, even at submicromolar concentrations (Supplementary Fig. S5b). The formation of the non-fluorescent lactone on aggregation becomes apparent from an analysis of the ultraviolet absorbance spectra of SiR-SNAP (Fig. 1c). In the absence of SDS the ultraviolet band at $290 \mathrm{~nm}$, characteristic for the lactone, is the major band, whereas the zwitterion with an absorbance maximum at $650 \mathrm{~nm}$ becomes predominant in the presence of SDS (Supplementary Fig. S5c). The formation of non-fluorescent spirolactones by SiR-carboxyl derivatives in the hydrophobic environment of aggregates is in agreement with their propensity to form spirolactones in solvents of relatively low dielectric constant. For example, SiR-SNAP is present predominantly as its spirolactone 

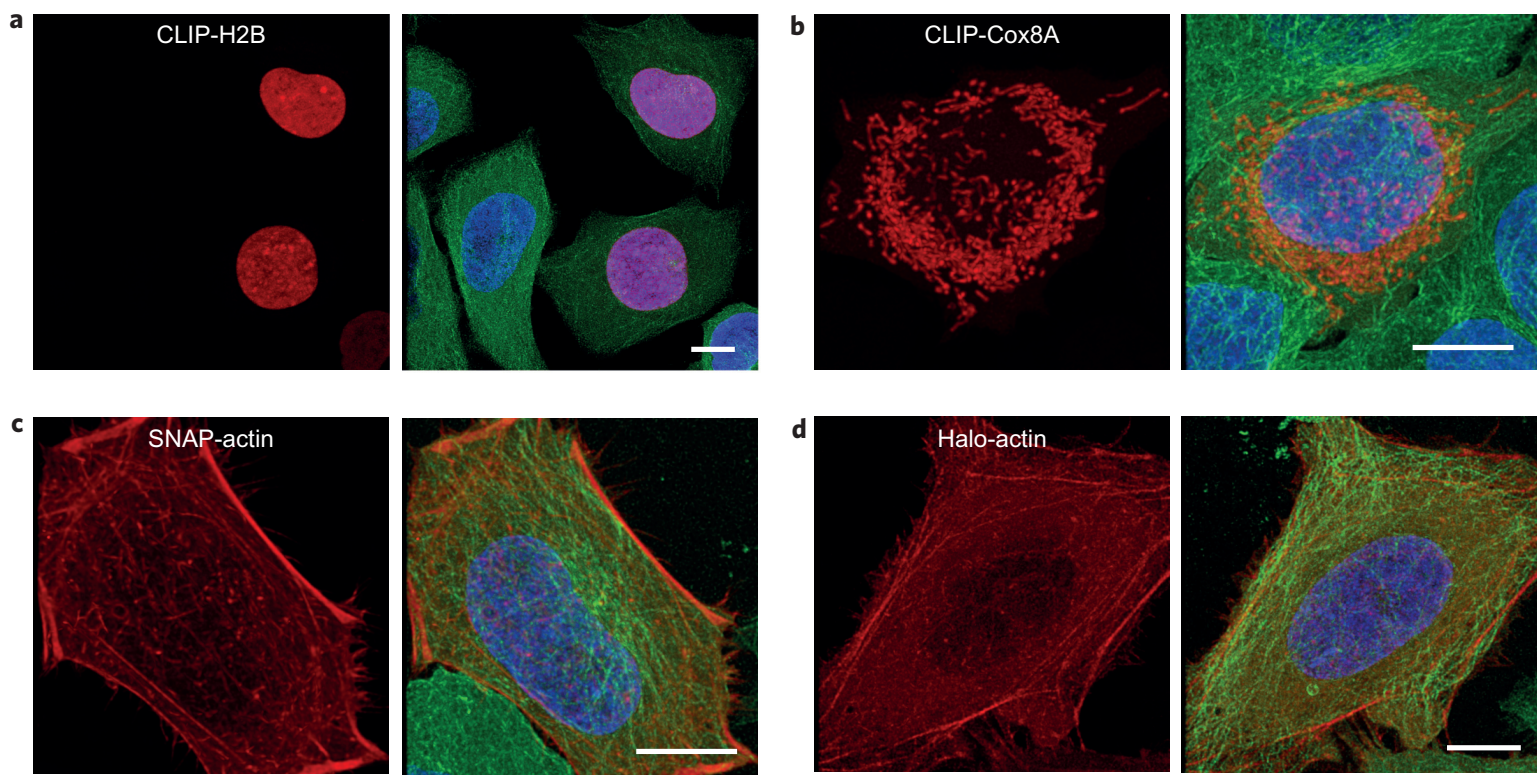

Figure 2 | Three-colour confocal fluorescence microscopy of the tagged proteins. a-d, SNAP (red in c), CLIP (red in a,b) and Halo-tagged proteins (red in d) in living HeLa cells expressing EGFP- $\alpha$-tubulin (green) and H2B-mCherry (blue) ${ }^{16}$. The characteristic staining of the fusion proteins demonstrates the suitability of SiR-carboxyl derivatives for live-cell imaging. Z-stacks of images were deconvolved using the Huygens Essentials package and presented as MIPs. Scale bar, $10 \mu \mathrm{m}$.

in ethanol ( $\varepsilon=25$, Fig. 1c), conditions under which the corresponding TMR derivative exists predominantly as a fluorescent zwitterion (Supplementary Fig. S5d). SiR-SNAP furthermore shows the same high propensity as SiR-carboxyl to form the spirolactone in dioxane-water mixtures (Fig. $1 \mathrm{~b}$ and Supplementary Fig. S5e). However, a difference between SiR-SNAP and SiR-carboxyl is that only SiR-SNAP aggregates and forms the spirolactone in pure water. Finally, a comparison of absorbance spectra of SiR-SNAP in the presence of SDS or after coupling to the SNAP-tag in the absence of SDS indicates that about $65 \%$ of SiR is present in its fluorescent zwitterionic form after covalent coupling to the SNAP-tag (Supplementary Fig. S5c). Overall, these experiments support a scenario in which coupling of SiR-carboxyl derivatives to protein tags favours formation of the fluorescent zwitterion, whereas aggregation of unreacted dye or its unspecific binding to hydrophobic surfaces favours formation of the non-fluorescent spirolactone, which thereby greatly facilitates imaging.

SiR-carboxyl derivatives thus permit imaging experiments with high sensitivity at a wavelength that shows little cellular autofluorescence and phototoxicity. An experiment that epitomizes the different attractive features of SiR-SNAP is a long-term imaging experiment in which cells that express SNAP fusions are grown in the presence of the probe and are imaged continuously over 48 hours (Supplementary Movie S1).

Another interesting application of SiR-carboxyl derivatives is their use for the labelling of intracellular proteins in more complex biological samples. We therefore investigated labelling of SNAP-tag in cortical neurons in rat-brain sections. For these experiments, SNAP-tag was expressed in cortical neurons through in utero electroporation of plasmids that encoded SNAP and GFP as markers (Fig. 3a). Three days after electroporation, brain sections were prepared, cultured and then incubated with SiR-SNAP. After removal of the excess SiR-SNAP through washing, the sample was fixed with paraformaldehyde and imaged (Fig. 3b). The images show specific labelling of cortical neurons that express SNAP-tag with no significant background fluorescence.

Live-cell super-resolution microscopy using SiR-SNAP. We also investigated the use of SiR-SNAP for live-cell super-resolution microscopy or nanoscopy of biological structures. All nanoscopy approaches rely on switching between the fluorescent and dark states of dyes ${ }^{17}$; thus, in such experiments, the photophysical properties of the fluorophore critically affect the attainable resolution $^{18}$. Furthermore, ideally nanoscopy of biological structures is performed on living cells, as this avoids the introduction of structural artefacts during fixation of the cells and permits characterization of the dynamic processes ${ }^{19}$.

One approach to nanoscopy relies on the stochastic conversion of isolated dyes into a fluorescent state, which thereby allows the subsequent reconstruction of super-resolved images from the positions of precisely localized single molecules ${ }^{20,21}$. Stochastic blinking of conventional fluorophores can be achieved by transiently transforming them into long-lived dark states, as realized in GSDIM (ground-state depletion followed by individual molecule return) ${ }^{22}$, direct stochastic optical reconstruction microscopy ((d)STORM $)^{23}$ and blinking microscopy ${ }^{24}$. To evaluate the potential of SiR-carboxyl derivatives for nanoscopy based on their stochastic blinking (dubbed as GSDIM/STORM here), we performed proofof-principle experiments with a SNAP-tag fusion protein of histone H2B. H2B-SNAP was expressed in U2OS cells, incubated with SiR-SNAP and imaged with a $640 \mathrm{~nm}$ laser power of $\sim 1 \mathrm{~kW} \mathrm{~cm}{ }^{-2}$. Under these conditions, a large percentage of the dye was maintained in a dark state, and a sparse population in the fluorescent state emitted, on average, 630 detectable photons, which allowed a high localization precision in each frame (Fig. 4). Blinking was very stable, with the number of molecules quickly stabilizing as the bleaching and recovery rates equilibrated (Supplementary Fig. S6). This allowed us to localize $\sim 140,000 \mathrm{~mol}-$ ecules in 10,000 frames, at a frame rate of $50 \mathrm{~Hz}$, to yield a superresolved image in just over three minutes. The dye's excellent photophysical properties permit GSDIM/STORM imaging to be repeated several times, as illustrated in Fig. 4c, where the same cell is imaged twice with a $10 \mathrm{~min}$ interval. Furthermore, SiR-labelled proteins can also be utilized for GSDIM/STORM on fixed cells (Supplementary Fig. S7). These experiments demonstrate that SiR-carboxyl derivatives are superior probes for live-cell GSDIM/STORM.

The excellent fluorescence properties of SiR-labelled proteins also make them attractive candidates for stimulated emission 

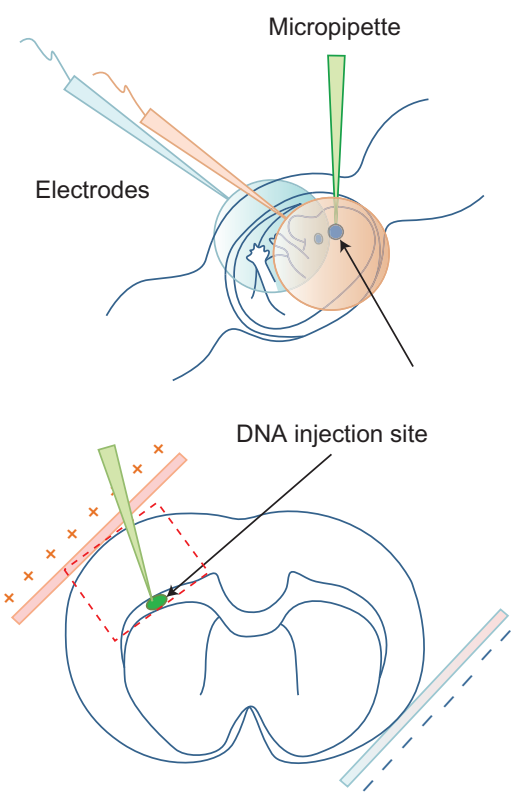
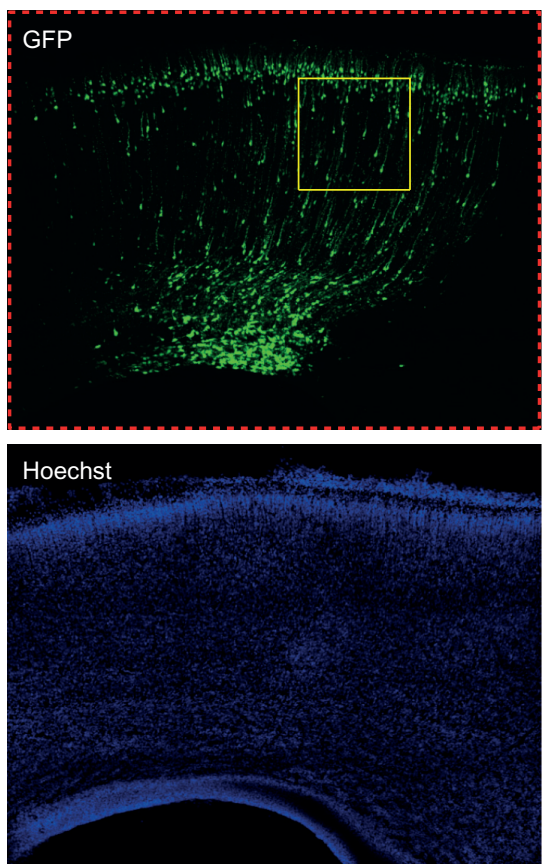

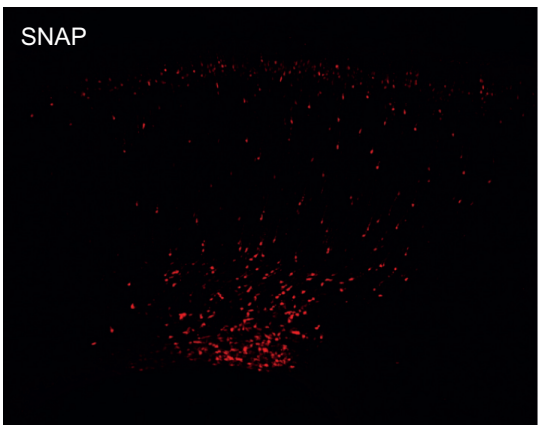

Merge

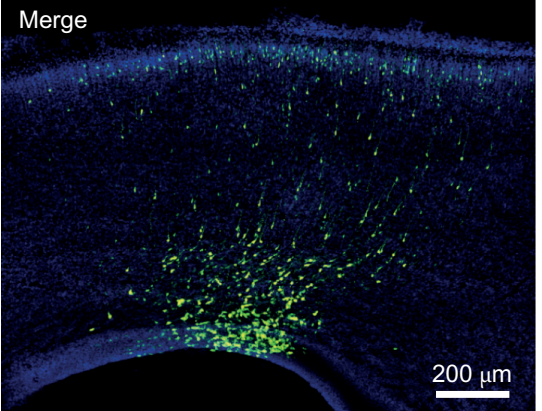

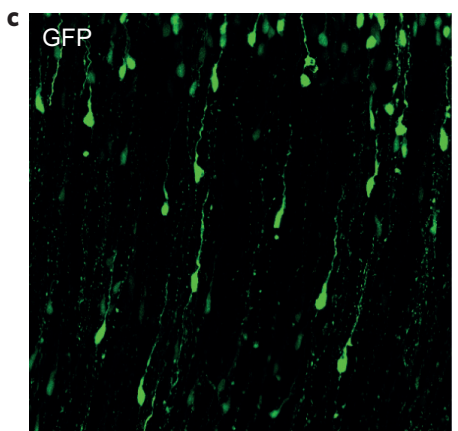
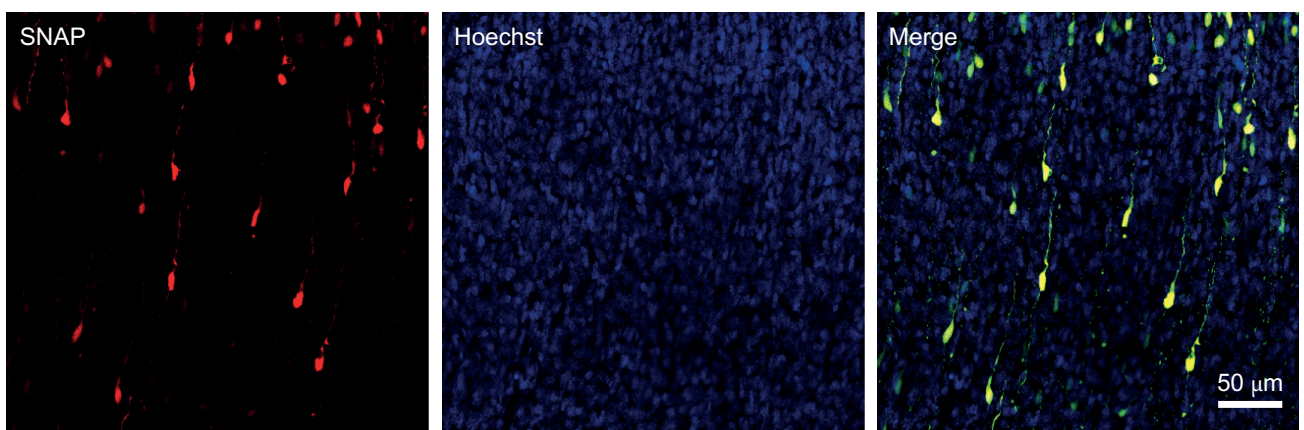

Figure 3 | Ex vivo labelling of a rat brain with SiR-SNAP. a, Scheme of in utero electroporation. Plasmid DNA is injected into an E16 rat embryo in utero through a micropipette and then electroporated with electrodes. The red square corresponds to the region shown in (b). b. SNAP and GFP plasmids (ratio 1:1) were introduced into a subset of neural progenitors at E16 by in utero electroporation. At E19, brains were sectioned and stained with SiR-SNAP and Hoechst. Scale bar, $200 \mu \mathrm{m}$. c, Images of the electroporated cortical neurons at a higher magnification (yellow box in b). The excellent overlap of the GFP and SiR-SNAP signals demonstrates the specificity of the labelling. Scale bar, $50 \mu \mathrm{m}$.
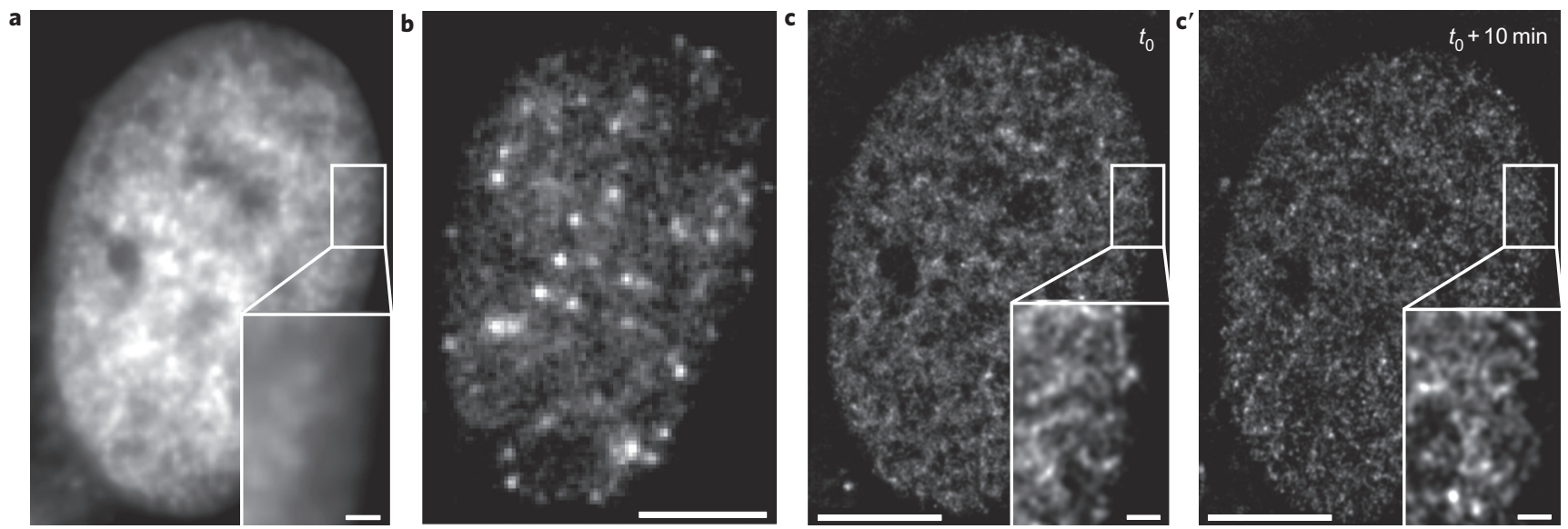

Figure 4 | Live-cell GSDIM/STORM imaging of nuclear localized H2B-SNAP-SiR. a, Wide-field image of H2B-SNAP-SiR does not allow the detection of substructures. $\mathbf{b}$, Single frame image showing the stochastic fluorescence (blinking) of individual molecules. c, GSDIM/STORM images reconstructed from 10,000 raw images ( $\mathbf{c}^{\prime}$ was taken ten minutes after $\mathbf{c}$ ). The enhancement in resolution permitted the detection of substructures. Scale bar, $5 \mu \mathrm{M}$ (inset, $500 \mathrm{~nm}$ ). 
a
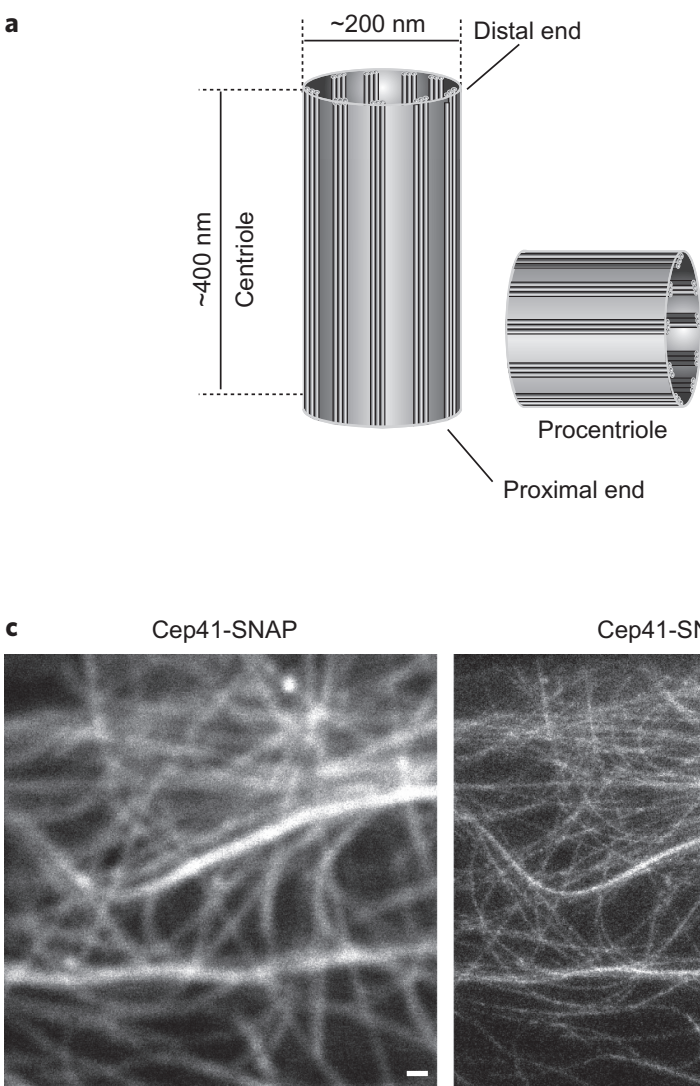

Confocal

d

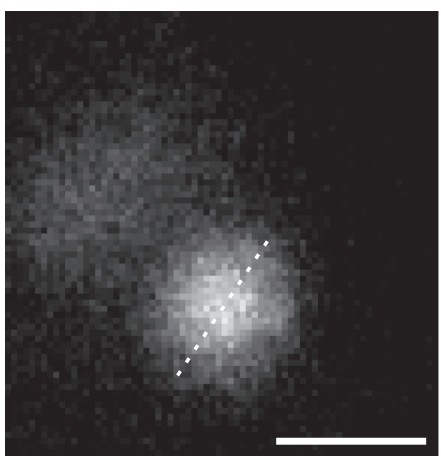

Confocal

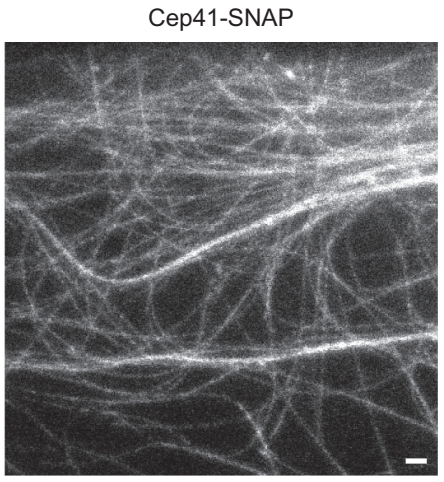

STED

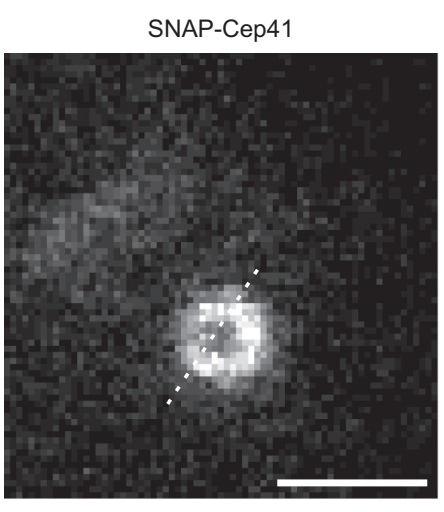

STED

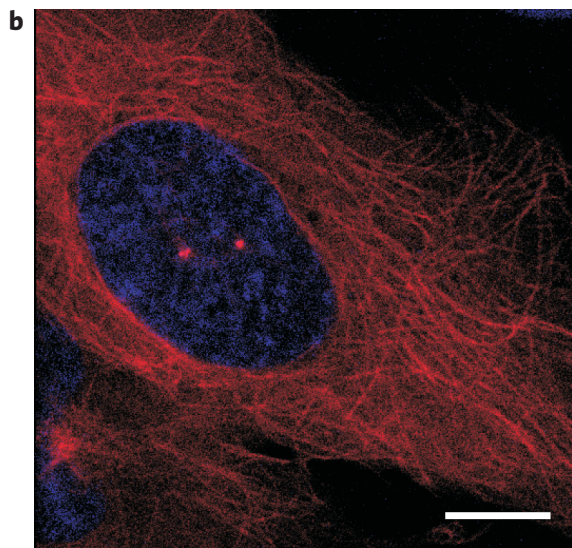

SNAP-Cep41/Hoechst 33342
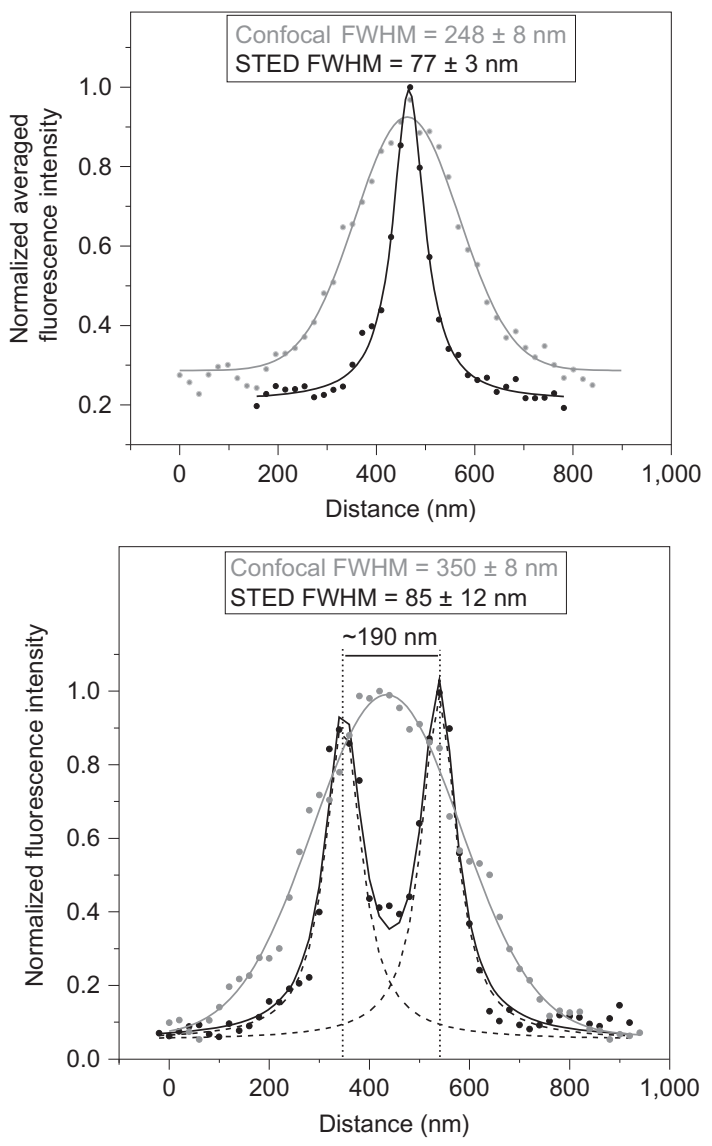

Figure 5 | Confocal and STED imaging of Cep41 protein localization in living U2OS cells. a, Schematic presentation of the centrosome structure. b, Confocal two-colour imaging of SNAP-Cep41 (red)-expressing cells stained with SiR-SNAP. Nuclear DNA was stained with Hoechst 33342 (blue). Scale bar, $10 \mu$ m. c, Comparison of confocal (left) and STED microscopy (middle) images of Cep41-SNAP bound to microtubules, along with an intensity line profile (right) obtained by averaging the profiles of seven different microtubule sections in the image. Scale bar, $500 \mathrm{~nm}$. d, Comparison of confocal (left) and STED (middle) microscopy images of SNAP-Cep41 localized at the centrosome with an intensity line profile (right) along the white dotted line marked in the images. The full width at half maximum (FWHM) of the imaged structures was obtained by fitting fluorescence-intensity profiles to Gauss or Lorentz distributions (OriginPro 8.1, http://www.originlab.com/). Two separated Lorentz distributions are indicated by grey dashed lines for the STED profile fitting. Distance between the peaks of the double Lorentz fitting was taken as the diameter of the structure. The diffuse signal visible in the top-left corner is the second centriole, which is located outside the focal plane. A corresponding two-colour image of SNAP-Cep41 and the centrosomal marker GFP-Centrin2 is presented in Supplementary Fig. S8. Scale bar, $500 \mathrm{~nm}$. Numbers are presented as the fitted value \pm standard error of the fit.

depletion (STED) microscopy ${ }^{25}$. In STED, the excitation laser is overlaid by a second STED laser that features at least one zero-intensity point and that restricts fluorescence emission, for example, only to the very focal centre. Scanning of such a reduced observation volume produces a fluorescence image with superior spatial resolution. STED microscopy is well suited for (dynamic) imaging in live cells ${ }^{26}$ and even in $v i v o^{27}$. Apart from a fluorescent protein with a limited fluorescence quantum yield and photostability ${ }^{28}$, no appropriate near-infrared fluorophores for STED microscopy of intracellular proteins in living cells are available currently. Therefore we investigated the potential of SiR-SNAP for live-cell STED microscopy of the centrosomal protein Cep41. The 


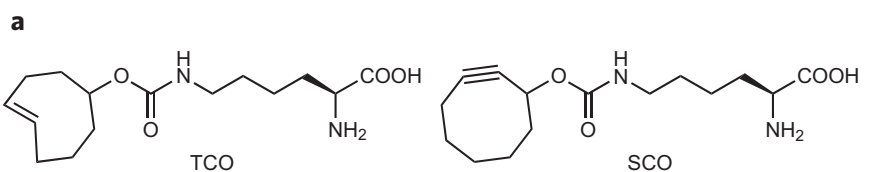

b

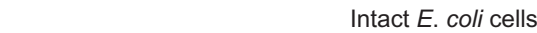

$+20 \mu \mathrm{M}$ SiR-tetrazine, $10 \mathrm{~min}$
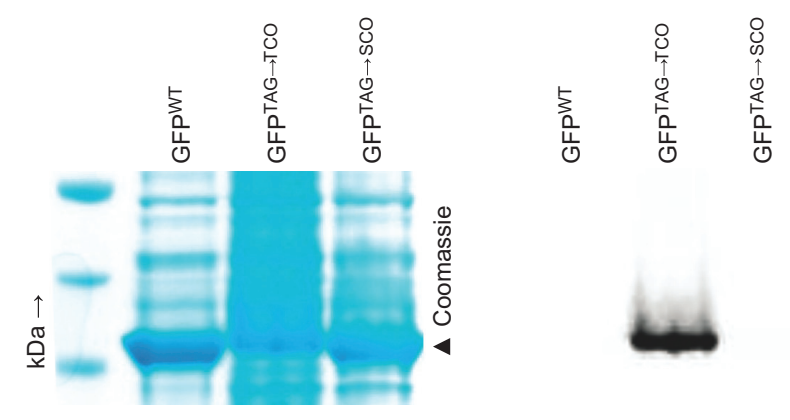

C
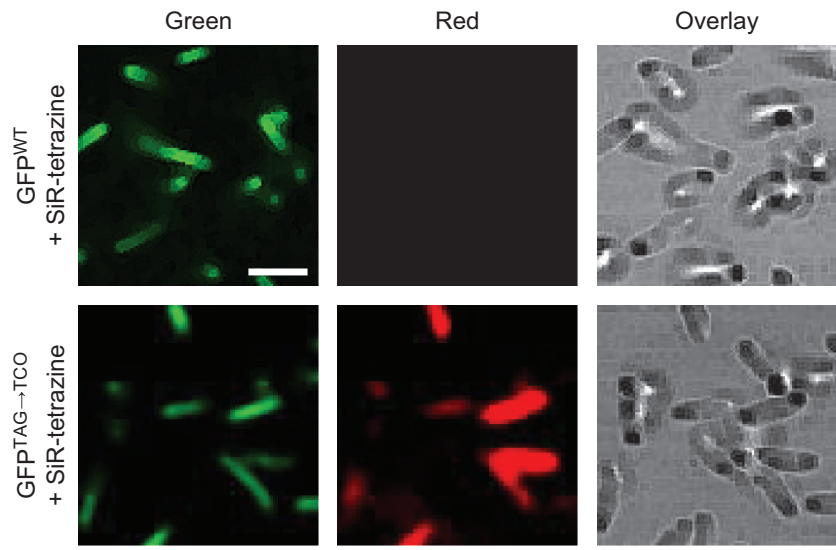

Figure 6 | Site-specific labelling of genetically encoded UAAs with SiR-tetrazine. a, Structures of UAA TCO and SCO. b, Intact E. coli cells expressing wild-type GFP (GFPWT), GFP ${ }^{T A G} \rightarrow$ TCO or GFP ${ }^{T A G} \rightarrow$ SCO were incubated with $20 \mu \mathrm{M}$ SiR-tetrazine for ten minutes and analysed via SDSPAGE. A fluorescent band ( $635 \mathrm{~nm}$ excitation) that indicates the successful labelling of GFP with SiR-tetrazine was observed only for GFP ${ }^{\mathrm{TAG} \rightarrow \mathrm{TCO} \text {, }}$ which confirms excellent bioorthogonality (black arrowhead indicates the height of the GFP). c, Live E. coli cells expressing GFPWT (upper row) and GFP $^{\text {TAG } \rightarrow \text { TCO }}$ (lower row) were incubated for ten minutes with $20 \mu \mathrm{M}$ SiR-tetrazine. After overnight washing, the cells were imaged for green (first column) or red (second column) fluorescence (third column shows the overlay of both channels with DIC image). Although GFP fluorescence was observed for all the samples, red fluorescence that originated from covalently reacted SiR-tetrazine was detected exclusively for GFP ${ }^{T A G} \rightarrow$ TCO (lower row), even though tRNA $\mathrm{A}^{\mathrm{Pyl}} / \mathrm{Py} \mathrm{AF}^{\mathrm{AF}}$ and TCO were also present during the expression in GFPWT cells (upper row). GFP ${ }^{T A G} \rightarrow$ SCO as an additional control is shown in Supplementary Fig. S9. Scale bar, $5 \mu \mathrm{m}$.

centrosome is the main microtubule-organizing centre of animal cells; it contains two microtubule-based centrioles of about $400 \mathrm{~nm}$ length and $200 \mathrm{~nm}$ diameter (Fig. 5a). The centrosome duplicates once per cell cycle, whereby a procentriole forms orthogonally at the proximal end of an existing centriole ${ }^{29-31}$. The precise localization of centrosomal proteins within the centriole by conventional microscopy is impossible because of its small size. Cep41 is reported to localize to interphase microtubules and centrosomes $^{32}$. Among other biological functions, mutations in Cep41 were linked to familial autism ${ }^{33}$ and Joubert syndrome ${ }^{34}$. For live-cell STED imaging experiments of Cep41, we constructed both $\mathrm{N}$ - and C-terminal SNAP-tagged Cep41 fusion proteins.
Labelling of SNAP-tag fusion proteins of Cep41 with SiR-SNAP in live U2OS cells and subsequent confocal microscopy confirmed the localization of SNAP-Cep41 to microtubules and centrosomes (Fig. 5b). Similar images were obtained for Cep41-SNAP on fixed cells (data not shown). Cep41-SNAP bound to microtubules was imaged by STED microscopy in live cells with a resolution far beyond that of confocal microscopy (Fig. 5c). Furthermore, we were able to record time-lapse movies of Cep41-SNAP bound to microtubules, which show the dynamics of such structures in a $10 \times 10 \mu \mathrm{m}^{2}$ field of view at a frame rate of $0.4 \mathrm{~Hz}$ (Supplementary Movie S2). We then investigated the precise localization of SNAP-Cep41 within centrosomes in living cells. Whereas live-cell confocal microscopy of SNAP-Cep41 labelled with SiR-SNAP did not show any particular structure beyond centrosomal localization of the Cep41, STED microscopy revealed that SNAP-Cep41 forms a ring-like structure along the axis of the centriole (Fig. 5d). The measured distance between intensity maxima of this ring-like structure is $175 \pm 13 \mathrm{~nm}$ $(N=7)$. This suggests that SNAP-Cep41 is bound (directly or indirectly) to the microtubules that form the wall of the centriole. Imaging of centrosomes oriented perpendicular to the imaging plane revealed the length of the structure formed by Cep41 to be $440 \pm 28 \mathrm{~nm} \quad(N=16)$ (Supplementary Fig. S8). This size is close to the reported length of centrioles and suggests that the ring-like structure of SNAP-Cep41 covers the entire length of the centriole. This model is further supported by two-colour colocalization experiments with GFP-Centrin2, a protein known to localize to the distal end of the centriole (Supplementary Fig. S8). To the best of our knowledge, no other centrosomal protein has been localized in living cells with a comparable resolution. The excellent spatial and temporal resolution achievable by a combination of SiR-SNAP and live-cell STED microscopy should become important for the investigation of numerous problems in biology.

Labelling of genetically encoded unnatural amino acids (UAAs) with SiR-tetrazine. To further expand the applications of SiRcarboxyl, we evaluated its coupling to intracellular proteins via the incorporation of UAAs in the proteins of interest and their subsequent specific fluorescence labelling ${ }^{35-37}$. Specifically, we investigated if a SiR-carboxyl derivative permits the fluorescence labelling of intracellular proteins that bear an appropriate UAA in Escherichia coli. The UAAs trans-cyclooctene lysine (TCO) and simple cyclooctyne lysine (SCO) (Fig. 6) were incorporated by amber (TAG) stop-codon suppression according to the expanded genetic code concept ${ }^{38}$. We used the previously reported tRNA $^{\text {Pyl }} /$ PylRS $^{\text {AF }}$ pair and GFP ${ }^{39 T A G}$ (TAG codon in position 39) in our study to express GFP $\mathrm{TAG}^{\mathrm{TAA}}$ and so genetically encode the respective $\mathrm{UAAs}^{36,39}$. The TCO derivative permits fast labelling of probes that carry tetrazines, whereas the SCO derivative serves as a control. A tetrazine was coupled to SiR-carboxyl and the resulting SiR-tetrazine was added to E. coli to express either $\mathrm{GFP}^{\mathrm{TAG} \rightarrow \mathrm{TCO}}$ or $\mathrm{GFP}^{\mathrm{TAG} \rightarrow \mathrm{SCO}}$. As shown in Fig. $6 \mathrm{~b}$ (and Supplementary Fig. S9), successful labelling of intact $E$. coli cells was obtained exclusively for $\mathrm{GFP}^{\mathrm{TAG} \rightarrow \mathrm{TCO}}$, but GFP ${ }^{\mathrm{TAG} \rightarrow \mathrm{SCO}}$, wild-type GFP $\left(\mathrm{GFP}^{\mathrm{WT}}\right)$ and any other protein in the $E$. coli cells did not show a red fluorescence signal. Fluorescence microscopy of live bacteria cells confirmed the results obtained via SDS-polyacrylamide gel electrophoresis (SDSPAGE): only $\mathrm{GFP}^{\mathrm{TAG} \rightarrow \mathrm{TCO}}$-expressing $E$. coli showed red fluorescence originating from covalently attached $\mathrm{SiR}$ (Fig. 6c). In contrast, $\mathrm{GFP}^{\mathrm{WT}}$-expressing bacteria that co-expressed $\mathrm{tRNA}^{\mathrm{Pyl}} / \mathrm{PylRS}^{\mathrm{AF}}$ and were also fed with TCO did not give any signal in the red channel. These proof-of-principle experiments thus demonstrate the general suitability of SiR-tetrazine for site-specific labelling of UAAs incorporated into proteins in the context of living cells. 


\section{Conclusions}

We introduce a near-infrared fluorophore ideally suited for the live-cell (super-resolution) microscopy of intracellular proteins. In addition to its excellent spectroscopic properties and membrane permeability, an important feature of the dye is its environmentally sensitive zwitterion-lactone equilibrium. This delicate equilibrium was exploited to generate fluorogenic probes for protein labelling with minimal background staining for different live-cell proteinlabelling approaches. We expect that these properties will stimulate the design of numerous other fluorescent probes based on this fluorophore and enable it to become an important tool in bioimaging.

Methods. Detailed procedures for the synthesis of all compounds, their characterization and the imaging experiments are given in the Supplementary Information.

Live-cell labelling and imaging. HeLa cells that expressed EGFP- $\alpha$-tubulin and H2B-mCherry ${ }^{16}$ were transfected with the appropriate construct, labelled and imaged in DMEM ${ }^{\text {gfp }}$ media (Evrogen) that contained 10\% FBS (Invitrogen). SNAP-tag, CLIP-tag and Halo-tag were labelled using 2.5, 3 and $1 \mu \mathrm{M}$ substrate $\left(0.5 \mathrm{~h}, 37^{\circ} \mathrm{C}\right)$, respectively. Imaging was performed in the presence or absence of the substrate. In the latter case, the substrate was removed by washing once with Hank's balanced salt solution (HBSS) or DMEM ${ }^{\mathrm{gfp}}$ media (Evrogen) containing $10 \%$ FBS (Invitrogen) (five minutes, room temperature). A Leica SP5 microscope equipped with a white light laser and HCX PL APO CS $63.0 \times 1.40$ oil ultraviolet objective was used. Excitation wavelengths were 490, 580 and $650 \mathrm{~nm}$ for GFP, mCherry and the SiR derivatives, respectively. Detection, using the Leica HyD (GaAsP hybrid detection system), of the GFP and SiR emission signals was set to ranges of 500-560 $\mathrm{nm}$ and $670-750 \mathrm{~nm}$, respectively. mCherry signals were detected with a regular photomultiplier tube detector in the 590-630 $\mathrm{nm}$ range. Voxel sizes $(69-96) \times(69-96) \times(126-256) \mathrm{nm}^{3}$ were selected depending on the size of the structure of interest. All three channels were imaged simultaneously using an $8,000 \mathrm{~Hz}$ resonance scanner with frame averaging of $10-12$ and one airy unit pinhole. Acquired images were deconvolved using the Huygens Essentials package. Images are presented as maximum intensity projections (MIPs).

U2OS cells that expressed SNAP-Cep41 were imaged using a Zeiss LSM 700 inverted microscope equipped with a Plan-Apochromat $63 \times 1.40$ oil DIC M27 objective. Cells were stained with $5 \mu \mathrm{M}$ SiR-SNAP and $0.1 \mu \mathrm{g} \mathrm{ml}^{-1}$ Hoechst 33342 (Sigma-Aldrich) $\left(0.5 \mathrm{~h}, 37^{\circ} \mathrm{C}\right)$, washed with HBSS (Lonza) (twice for five minutes, room temperature) and imaged in the same buffer. Two-colour simultaneous acquisitions (pixel size $100 \times 100 \mathrm{~nm}^{2}$, line averaging of eight) were used, with $405 \mathrm{~nm}$ and $640 \mathrm{~nm}$ excitation lasers in combination with a $555 \mathrm{~nm}$ short pass (Hoechst channel) and a $640 \mathrm{~nm}$ long pass (SiR channel) emission filters.

In vivo labelling and imaging. We used the in utero electroporation method to deliver expression constructs into rat cortical neurons, as described previously ${ }^{40}$. Briefly, uterine horns of pregnant rats at E16 were exposed and 1-2 $\mu \mathrm{g}$ of plasmid solution $(\mathrm{GFP} / \mathrm{SNAP}=1: 1)$ was injected into the lateral ventricle of the embryonic brain with a fine-glass micropipette. Embryos were then clamped between $5 \mathrm{~mm}$ diameter tweezers-type disc electrodes (CUY650-5, Tokiwa Science) and were given five electrical pulses $(55 \mathrm{~V}, 100 \mathrm{~ms})$ with an interval of one second (electroporator ECM830, BTX). The uterine horns were then placed back into the abdominal cavity to allow the embryos to continue development. At E19, embryos were taken out and brain sections were made with a vibratome at a thickness of $100 \mu \mathrm{m}$. Live brain section were cultured in a standard complete culture medium $(\mathrm{DMEM} / \mathrm{F} 12 / \mathrm{FBS}=8: 1: 1$; where $\mathrm{F} 12=$ nutrient mixture F12). The brain sections were then stained with a $10 \mu \mathrm{M}$ SiR-SNAP substrate (two hours, $37^{\circ} \mathrm{C}$ ). After the labelling, brain sections were washed with PBS buffer (three times for 30 minutes) and then kept in a cultured medium overnight (at $37^{\circ} \mathrm{C}$ ). Before imaging, the brain sections were fixed with $4 \%$ paraformaldehyde (20 minutes, room temperature) and stained with Hoechst (15 minutes, room temperature). Images were then captured using a Nikon Ti image system and analysed with ImageJ software.

GSDIM/STORM imaging of living cells. U2OS cells were cultured in DMEM with GlutaMAX-1 (Invitrogen) supplemented with 10\% FBS in a humidified incubator $\left(5 \% \mathrm{CO}_{2}\right.$ at $\left.37^{\circ} \mathrm{C}\right)$. Cells were plated on $25 \mathrm{~mm}$ diameter, $1 \mathrm{~mm}$ thickness coverslips (Menzel) 48 hours before imaging. Transient transfection of SNAPf-H2B ( $2 \mu \mathrm{g}$ (deoxy)ribonucleic acid (DNA) mixed with $3 \mu$ l Fugene6, Roche) was performed 24 hours before imaging. Cells were stained for one hour with 500 nM SiR-SNAP in DMEM, rinsed three times in phenol red-free DMEM (twice for five minutes and once for one hour) and 10,000 frames at $50 \mathrm{~Hz}$ were recorded in Leibovitz's medium (Invitrogen) at room temperature $\left(24^{\circ} \mathrm{C}\right)$.

The raw data were first filtered by using convolution with a zero-sum Gaussian, and then single molecules were localized using the image $(\mathrm{NIH}, \mathrm{http}: / /$ imagej.nih. gov/ij/) plugin Octane ${ }^{41}$. Each localized molecule was rendered as a $40 \mathrm{~nm}$ wide Gaussian spot (except tubulin images, for which it was $15 \mathrm{~nm}$ ) to construct the final GSDIM/STORM image.
Live-cell STED microscopy. U2OS cells that expressed the appropriate SNAP fusion were seeded on glass coverslips. Cells were incubated in H-DMEM with 10\% FBS that contained $2.5 \mu \mathrm{M}$ SiR-SNAP $\left(30\right.$ minutes, $37^{\circ} \mathrm{C}$ ). The medium was changed to fresh H-DMEM with $10 \%$ FBS and imaging was performed on a home-built STED microscope described in detail previously ${ }^{42}$ and expanded by a fast-beam scanner and a blue-excitation laser for simultaneous detection of the GFP fluorescence (in a normal diffraction-limited confocal mode). Image acquisition was controlled by the software ImSpector (www.imspector.de) and by an analogue input/output card. The excitation intensity was in the range $5-8 \mu \mathrm{W}$ at a diffraction-limited focal diameter of approximately $250 \mathrm{~nm}$. The STED power measured in the back aperture of the objective was $180 \mathrm{~mW}$. The resolution was approximately $50 \mathrm{~nm}$, as measured independently by imaging $20 \mathrm{~nm}$ large fluorescent beads.

Site-specific labelling of genetically encoded UAAs in $\boldsymbol{E}$. coli. Cell pellets of E. coli that expressed GFP ${ }^{\mathrm{WT}}, \mathrm{GFP}^{\mathrm{TAG} \rightarrow \mathrm{TCO}}$ or $\mathrm{GFP}^{\mathrm{TAG} \rightarrow \mathrm{SCO}}$ were resuspended in $1 \times$ PBS buffer and the optical density $600\left(\mathrm{OD}_{600}\right)$ was adjusted to $\sim 4$. A total volume of $400 \mu \mathrm{l}$ of cell suspension $\left(\mathrm{OD}_{600} \sim 4\right)$ was then incubated with $20 \mu \mathrm{M}$ SiR-tetrazine ( $10 \mathrm{mM}$ stock solution in DMSO) at $37^{\circ} \mathrm{C}$ in the dark and shaken for ten minutes. Cells were pelleted and washed twice with $1 \times$ PBS before overnight incubation at $4{ }^{\circ} \mathrm{C}$ in $1 \times$ PBS that contained 5\% DMSO to wash out excess dye. The cells were washed again twice with $1 \times$ PBS before they were allowed to settle on a coverslip. Cells were then mounted on a Leica SP2 confocal microscope employing a $63 \times$ oil, 1.4 numerical aperture objective (Leica, Mannheim, Germany). Images that contained $512 \times 512$ pixels were acquired at a scan speed of $400 \mathrm{~Hz}$ and a zoom factor of four to yield a final pixel size of $116.3 \mathrm{~nm} \times 116.3 \mathrm{~nm}$. In addition to a differential interference contrast (DIC) image the sample was sequentially excited using a $488 \mathrm{~nm}$ and $633 \mathrm{~nm}$ laser along with recording the fluorescence signal in the green $(500-550 \mathrm{~nm})$ or red $(655-720 \mathrm{~nm})$ channel, respectively.

Received 21 August 2012; accepted 28 November 2012; published online 6 January 2013

\section{References}

1. Hinner, M. \& Johnsson, K. How to obtain labeled proteins and what to do with them. Curr. Opin. Biotechnol. 21, 766-776 (2010).

2. Schneckenburger, H. et al. Light exposure and cell viability in fluorescence microscopy. J. Microsc. 245, 311-318 (2012).

3. Pellett, P. A. et al. Two-color STED microscopy in living cells. Biomed Opt Express 2, 2364-2371 (2011).

4. Wombacher, R. et al. Live-cell super-resolution imaging with trimethoprim conjugates. Nature Methods 7, 717-719 (2010).

5. Jones, S. A., Shim, S. H., He, J. \& Zhuang, X. Fast, three-dimensional super-resolution imaging of live cells. Nature Methods 8, 499-508 (2011).

6. van de Linde, S., Heilemann, M. \& Sauer, M. Live-cell super-resolution imaging with synthetic fluorophores. Annu. Rev. Phys. Chem. 61, 519-540 (2012).

7. Koide, Y., Urano, Y., Hanaoka, K., Terai, T. \& Nagano, T. Evolution of group 14 rhodamines as platforms for near-infrared fluorescence probes utilizing photoinduced electron transfer. ACS Chem. Biol. 6, 600-608 (2011)

8. Egawa, T. et al. Development of a far-red to near-infrared fluorescence probe for calcium ion and its application to multicolor neuronal imaging. J. Am. Chem. Soc. 133, 14157-14159 (2011).

9. Koide, Y., Urano, Y., Hanaoka, K., Terai, T. \& Nagano, T. Development of an Si-rhodamine-based far-red to near-infrared fluorescence probe selective for hypochlorous acid and its applications for biological imaging. J. Am. Chem. Soc. 133, 5680-5682 (2011).

10. Keppler, A. et al. A general method for the covalent labeling of fusion proteins with small molecules in vivo. Nature Biotechnol. 21, 86-89 (2003).

11. Keppler, A., Pick, H., Arrivoli, C., Vogel, H. \& Johnsson, K. Labeling of fusion proteins with synthetic fluorophores in live cells. Proc. Natl Acad. Sci. USA 101, 9955-9959 (2004).

12. Wang, T. et al. Spirolactonized Si-rhodamine: a novel NIR fluorophore utilized as a platform to construct Si-rhodamine-based probes. Chem. Commun. 48, 8781-8783 (2012)

13. Åkerlöf, G. \& Short, A. O. The dielectric constant of dioxane-water mixtures between 0 and $80^{\circ}$. J. Am. Chem. Soc. 58, 1241-1243 (1936).

14. Gautier, A. et al. An engineered protein tag for multiprotein labeling in living cells. Chem. Biol. 15, 128-136 (2008).

15. Los, G. V. et al. HaloTag: a novel protein labeling technology for cell imaging and protein analysis. ACS Chem. Biol. 3, 373-382 (2008).

16. Held, M. et al. CellCognition: time-resolved phenotype annotation in high-throughput live cell imaging. Nature Methods 7, 747-754 (2010).

17. Hell, S. W. Microscopy and its focal switch. Nature Methods 6, 24-32 (2009).

18. Dempsey, G. T., Vaughan, J. C., Chen, K. H., Bates, M. \& Zhuang, X. Evaluation of fluorophores for optimal performance in localization-based super-resolution imaging. Nature Methods 8, 1027-1036 (2011).

19. Schnell, U., Dijk, F., Sjollema, K. A. \& Giepmans, B. N. Immunolabeling artifacts and the need for live-cell imaging. Nature Methods 9, 152-158 (2012).

20. Rust, M. J., Bates, M. \& Zhuang, X. Sub-diffraction-limit imaging by stochastic optical reconstruction microscopy (STORM). Nature Methods 3, 793-795 (2006). 
21. Betzig, E. et al. Imaging intracellular fluorescent proteins at nanometer resolution. Science 313, 1642-1645 (2006).

22. Folling, J. et al. Fluorescence nanoscopy by ground-state depletion and single-molecule return. Nature Methods 5, 943-945 (2008).

23. Heilemann, M. et al. Subdiffraction-resolution fluorescence imaging with conventional fluorescent probes. Angew. Chem. Int. Ed. 47, 6172-6176 (2008)

24. Steinhauer, C., Forthmann, C., Vogelsang, J. \& Tinnefeld, P. Superresolution microscopy on the basis of engineered dark states. J. Am. Chem. Soc. 130, 16840-16841 (2008).

25. Hell, S. W. \& Wichmann, J. Breaking the diffraction resolution limit by stimulated emission: stimulated-emission-depletion fluorescence microscopy. Opt. Lett. 19, 780-782 (1994).

26. Hein, B. et al. Stimulated emission depletion nanoscopy of living cells using SNAP-tag fusion proteins. Biophys. J. 98, 158-163 (2010).

27. Berning, S., Willig, K. I., Steffens, H., Dibaj, P. \& Hell, S. W. Nanoscopy in a living mouse brain. Science 335, 551 (2012)

28. Morozova, K. S. et al. Far-red fluorescent protein excitable with red lasers for flow cytometry and superresolution STED nanoscopy. Biophys. J. 99, L13-L15 (2010).

29. David, R. Cell cycle: building the centriole. Nature Rev. Mol. Cell. Biol. 12, 342 (2011).

30. Azimzadeh, J. \& Marshall, W. F. Building the centriole. Curr. Biol. 20, R816-R825 (2010).

31. Bettencourt-Dias, M. \& Glover, D. M. Centrosome biogenesis and function: centrosomics brings new understanding. Nature Rev. Mol. Cell. Biol. 8, 451-463 (2007).

32. Gache, V. et al. Xenopus meiotic microtubule-associated interactome. PLoS One 5, e9248 (2010).

33. Korvatska, O. et al. Mutations in the TSGA14 gene in families with autism spectrum disorders. Am. J. Med. Genet. B Neuropsychiatr. Genet. 156, 303-311 (2011).

34. Lee, J. E. et al. CEP41 is mutated in Joubert syndrome and is required for tubulin glutamylation at the cilium. Nature Genet. 44, 193-199 (2012).

35. Lang, K. et al. Genetically encoded norbornene directs site-specific cellular protein labelling via a rapid bioorthogonal reaction. Nature Chem. 4, 298-304 (2012).

36. Plass, T. et al. Amino acids for Diels-Alder reactions in living cells. Angew. Chem. Int. Ed. 51, 4166-4170 (2012).

37. Lang, K. et al. Genetic encoding of bicyclononynes and trans-cyclooctenes for site-specific protein labeling in vitro and in live mammalian cells via rapid fluorogenic Diels-Alder reactions. J. Am. Chem. Soc. 134, 10317-10320 (2012).

38. Liu, C. C. \& Schultz, P. G. Adding new chemistries to the genetic code. Annu. Rev. Biochem. 79, 413-444 (2010).
39. Plass, T., Milles, S., Koehler, C., Schultz, C. \& Lemke, E. A. Genetically encoded copper-free click chemistry. Angew. Chem. Int. Ed. 50, 3878-3881 (2011).

40. Saito, T. \& Nakatsuji, N. Efficient gene transfer into the embryonic mouse brain using in vivo electroporation. Dev. Biol. 240, 237-246 (2001).

41. Niu, L. \& Yu, J. Investigating intracellular dynamics of FtsZ cytoskeleton with photoactivation single-molecule tracking. Biophys. J. 95, 2009-2016 (2008).

42. Mueller, V. et al. STED nanoscopy reveals molecular details of cholesteroland cytoskeleton-modulated lipid interactions in living cells. Biophys. J. 101, 1651-1660 (2011).

\section{Acknowledgements}

This work was supported by the Swiss National Science Foundation, the Chemica National Centre of Competence in Research Biology, European Research Council grant no. 243016-PALMassembly and the École Polytechnique Fédérale de Lausanne. G.L. was supported by a Federation of European Biochemical Societies long-term fellowship. K.U. was supported by a Grant-in-Aid for Postdoctoral Fellowships for Foreign Researchers Fellows. C.S. is supported by TRR83, and T.P. by the Fonds der Chemischen Industrie. E.A.L. acknowledges funding from the Emmy Noether program of the Deutsche Forschungsgemeinschaft. E.A.L. and C.S. also acknowledge funding from the SPP 1623 of the Deutsche Forschungsgemeinschaft. The authors thank A. Schena, B. Mollwitz and P. Gönczy for sharing reagents and cell lines, S. Hell and S. Jakobs (MPI Göttingen) for excellent support, Tanja Gilat (Max-Planck Institut (MPI) Göttingen) for preparation of the cells and A. Schönle (MPI Göttingen) for support with the software ImSpector.

\section{Author contributions}

All authors planned the experiments and co-wrote the paper. K.U. designed the structure of SiR-carboxyl. K.U., L.R. and I.C. performed the chemical syntheses. G.L., K.U. and L.R. characterized the dyes. G.L., A.H. and V.M. performed the confocal and STED microscopy with subsequent data analysis. N.O. and S.M. performed the GSDIM/STORM imaging and data analysis. T.P., C.S. and E.A.L performed the amber suppression experiments and analysis. G.Y., Z-G.L. and P.H. performed the labelling in brain sections.

\section{Additional information}

Supplementary information and chemical compound information are available in the online version of the paper. Reprints and permission information is available online at http://www.nature.com/reprints. Correspondence and requests for materials should be addressed to K.J.

\section{Competing financial interests}

The authors declare no competing financial interests. 